Short Review: Open Access

\title{
Selection of a Progress Monitoring Instrument for Substance Use Disorder Treatment
}

\author{
Espen Ajo Arnevik*
}

Norwegian National Advisory unit on Substance use disorder Treatment, University of Oslo, Norway

*Corresponding author: Espen Ajo Arnevik, Head of research, Associate Professor, Norwegian National Advisory unit on Substance use disorder Treatment, University of Oslo, Norway, Europe, E-mail: ESARNE@ous-hf.no

\begin{abstract}
Recent research shows the value of ensuring the quality and efficiency of treatment using progress monitoring instruments. Before implementing progress monitoring instruments in clinical practice, health managers and clinicians must decide which particular instrument to use. Most identified progress monitoring instruments for substance use disorder treatment seems to include symptom level, relational functioning and social functioning, and all report adequate psychometric qualities. Of concern is that central variables such as alliance, user satisfaction and motivation most often are neglected. There is a need to validate existing instruments used in mental health treatments for substance use disorder populations. In addition, specialized progress monitoring instruments for substance use disorder populations should continue to be developed, based on the needs of clinicians and health managers, and on previous research.
\end{abstract}

\section{Keywords}

Progress monitoring, Substance use disorder, Treatment

\section{Background}

Monitoring of treatment is standard practice for many medical conditions. The use of progress monitoring (PM) instruments in mental health treatment is increasing, and several organizations (e.g., American Psychiatric Association) and countries (e.g., Australia and Switzerland) recommend such instruments as a means for ensuring the quality and efficiency of treatment. In addition, PM is a means for personalizing treatment and increasing patient involvement. Substance use disorder (SUD) treatment has been slow to adopt this practice. In a consultative document for the proposed Norwegian guidelines for SUD treatment [1], the first recommendation proclaims that: "To ensure customized treatment systemized and continuous monitoring of treatment progress should be facilitated" (our translation). The challenge for leaders and clinicians faced with the implementation of the new guidelines is to decide which of the available PM instruments to use.

\section{Progress Monitoring (PM) in Substance Use Disorder} (SUD) treatment

Many measurements and feedback interventions have been evaluated in mental health treatment [2]. Historically, urine drug screens and breathalysers have been commonly used as PM instruments used in SUD treatment. In addition, drop-out or attendance in treatment has been seen as an indication of negative or positive treatment progress, respectively [3]. Positive drug screens or missed sessions would indicate lack of progress in treatment, perhaps leading to a change in the treatment plan or even expulsion from treatment. More recently, specific measures have been developed to assess progress in the treatment of SUD.

A recent review of PM in mental health and SUD treatment concluded that previous meta-analyses showed PM significantly improved the outcome in mental health treatment, and focused on the need for further research and the adoption of PM methods in SUD treatment [2]. In addition to the eight PM instruments reviewed in the paper, the Session Rating Scale (SRS) [4], the Outcome Rating Scale (ORS) [5] and the OQ-45.2 [6], have been used in SUD treatment and are under ongoing study at different study sites in Norway and internationally. There might be some local instruments in use that have not yet come to our attention.

From a clinician or leaders view, a successful PM instrument should balance ease of administration, to allow for routine use, with comprehensiveness, to capture multiple domains of importance. Items should reflect constructs or variables that are linked to patient problems, and psychosocial and relational issues that are linked to service needs, to predict possible iatrogenic effects and outcomes. In addition, the instruments should provide a snapshot that depicts the patient's status, and create a cumulative record of the patient's progress over time. Finally, the instruments should have a user interface suitable for the patient group in question.

None of the PM instruments reviewed in the paper fulfil all of the criteria (Table 1). All the instruments seem to have adequate psychometric qualities. Most of them also include variables such as symptom level, relational functioning and social functioning. The threshold for use seems to be at an acceptable level, although many of the instruments are not available in Scandinavian languages (there might be local translations of the instruments that I did not find by searching the Internet). On the other hand, central variables such as alliance, user satisfaction and motivation are generally neglected in the reviewed instruments. This lack of focus in the development of the PM instruments is surprising, given the amount of research literature supporting the importance of alliance and motivation as effective and necessary ingredients in achieving good treatment results and reducing drop-out [3]. Although the effect of user 
satisfaction on treatment effect is still under debate, there is no doubt that we have an ethical responsibility regarding user involvement. In using PM instruments, we have a unique opportunity to ask for user satisfaction in the treatment process in a structured way, and to use it to make decisions about changes in treatment, together with the patient.

Only three of the instruments allows for a test frequency of one week or every session. The others vary between one month, and the start and end of treatment. A PM instrument is supposed to monitor the treatment process, be able to detect possible iatrogenic effects and prevent possible drop-out; one could question whether these instruments were intended to be PM instruments, or designed more as outcome measures.

Research on using PM instruments designed for mental health services on SUD populations is scarce, but there have been some steps in establishing a research base for PM in SUD treatment $[15,16]$. The demographics of SUD populations tend to differ from those in mental health studies, and although the studies suggest potential for PM instruments to help retain patients longer in treatment, more research needs to be done.

\section{Conclusion}

It appears that the three PM instruments originally designed for mental health treatment (SRS, ORS, OQ-45.2) are the most closely aligned to the concept of PM. These instruments fulfil most of the criteria determined by the review group. One could envision three scenarios to take PM in SUD treatment a step further: 1) further research on the SRS, ORS and OQ-45.2;2) review of more of the PM instruments developed for mental health treatment, with research to determine whether they can be used in SUD populations; and 3) the development of specialized PM instruments for SUD populations. Currently, an ongoing focus on the SRS, ORS and OQ-45, and a parallel process to develop specialized PM instruments for SUD populations, based on the needs of clinicians and health managers, and on previous research seems to be the most promising strategy regarding the state of the research field in SUD treatment and PM instruments.

\section{Author's Contribution}

The author wrote the manuscript and approved the manuscript.

\section{Acknowledgments}

Thank you to Gro Syversen, Rune Aaen Jørgensen, Christian Aarup Skeie, Ingrid Benneche, Anne T Brochmann, Ståle Alstadius, Hanne H. Brorson, Kari Bakke Larring and Hilde Harwiss for their contributions.

\section{References}

1. https://helsedirektoratet.no/retningslinjer.

2. Goodman JD, McKay JR, DePhilippis D (2013) Progress monitoring in mental health and addiction treatment: A means of improving care. Professional Psychology: Research and Practice 44: 231-246.

3. Brorson HH, Ajo Arnevik E, Rand-Hendriksen K, Duckert F (2013) Drop-out from addiction treatment: a systematic review of risk factors. Clin Psychol Rev 33: 1010-1024.

4. Duncan BL, Sparks SD, Claud DA, Reynolds LR, Brown J, et al. (2003) The Session Rating Scale: Preliminary psychometric properties of a "working" alliance measure. Journal of brief therapy 3: 3-12.

5. Miller SD, Duncan BL, Brown J, Sparks JA, Claud DA (2003) The Outcome Rating Scale: A preliminary study of the reliability, validity, and feasability of a brief visual analog measure. Journal of brief therapy 2: 91-100.

6. Lambert MJ, Morton JJ, Hatfield DR, Harmon C, Hamilton S, et al. (2004) Administration and scoring manual for the OQ-45.2 Outcome Questionnaire. OQ measures, Salt Lake City, USA.

7. Pulford J, Deering DE, Robinson G, Wheeler A, Adamson SJ, et al. (2010) Development of a routine outcome monitoring instrument for use with clients in the New Zealand alcohol and other drug treatment sector: The Alcohol and Drug Outcome Measure (ADOM). New Zealand J Psychol 39: 35-45.

8. Butler SF, Budman SH, McGee MD, Davis MS, Cornelli R, et al. (2005) Addiction severity assessment tool: development of a self-report measure for clients in substance abuse treatment. Drug Alcohol Depend 80: 349-360.

9. Simpson M, Lawrinson P, Copeland J, Gates P (2009) The alcohol treatment outcome measure (ATOM): A new clinical tool for standardising outcome measurement for alcohol treatment. Addict Behav 34: 121-124.

10. Cacciola JS, Alterman Al, Dephilippis D, Drapkin ML, Valadez C Jr, et al. (2013) Development and initial evaluation of the Brief Addiction Monitor (BAM). J Subst Abuse Treat 44: 256-263.

11. Lawrinson P, Copeland J, Indig D (2005) Development and validation of a brief instrument for routine outcome monitoring in opioid maintenance pharmacotherapy services: The brief treatment outcome measure (BTOM). Drug Alcohol Depend 80: 125-133.

12. Marsden J, Gossop M, Stewart D, Best D, Farrell M, et al. (1998) The Maudsley Addiction Profile (MAP):A brief instrument for assessing treatment outcome. Addiction 93: 1857-1868.

13. Deering DE, Sellman JD, Adamson SJ, Horn J, Frampton CM (2008) Development of a brief treatment instrument for routine clinical use with methadone maintenance treatment clients: The Methadone Treatment Index. Subst Use Misuse 43: 1666-1680.

14. Marsden J, Farrell M, Bradbury C, Dale-Perera A, Eastwood B, et al. (2008) Development of the treatment outcomes profile. Addiction 103: 1450-1460.

15. Crits-Christoph P, Ring-Kurtz S, Hamilton JL, Lambert MJ, Gallop R, et al. (2012) A preliminary study of the effects of individual patient-level feedback in outpatient substance abuse treatment programs. J Subst Abuse Treat 42: 301-309.

16. Miller SD, Duncan BL, Sorrell R, Brown GS (2005) The partners for change outcome management system. J Clin Psychol 61: 199-208. 